\title{
A note on VNP-completeness and border complexity
}

\author{
Christian Ikenmeyer \\ University of Liverpool \\ christian.ikenmeyer@liverpool.ac.uk
}

Abhiroop Sanyal
Chennai Mathematical Institute
abhiroop.sanyal@gmail.com

\begin{abstract}
In 1979 Valiant introduced the complexity class VNP of p-definable families of polynomials, he defined the reduction notion known as p-projection and he proved that the permanent polynomial and the Hamiltonian cycle polynomial are VNP-complete under p-projections.

In 2001 Mulmuley and Sohoni (and independently Bürgisser) introduced the notion of border complexity to the study of the algebraic complexity of polynomials. In this algebraic machine model, instead of insisting on exact computation, approximations are allowed. This gives VNP the structure of a topological space. In this short note we study the set VNPC of VNP-complete polynomials. We show that the complement VNP \VNPC lies dense in VNP. Quite surprisingly, we also prove that VNPC lies dense in VNP. We prove analogous statements for the complexity classes VF, VBP, and VP.

The density of VNP \VNPC holds for several different reduction notions: p-projections, border pprojections, c-reductions, and border c-reductions. We compare the relationships of the completeness notions under these reductions and separate most of the corresponding sets. Border reduction notions were introduced by Bringmann, Ikenmeyer, and Zuiddam (JACM 2018). Our paper is the first structured study of border reduction notions.
\end{abstract}

2012 ACM Subject Classification Theory of computation $\rightarrow$ Algebraic complexity theory

Keywords and phrases algebraic complexity theory, VNP, border complexity, reductions, completeness, topology

Funding Christian Ikenmeyer: CI supported by DFG grant IK 116/2-1.

Acknowledgements We thank Michael Forbes for helpful insights about the relative power of reduction notions. Moreover, anonymous referees made us generalize our two main theorems. We thank Josh Grochow for discussions that led to significant adjustments in Section 2.2.

\section{Introduction}

Valiant's famous determinant versus permanent conjecture [39] states that the algebraic complexity class VBP (polynomials that can be written as determinants of polynomially large matrices of linear polynomials) is strictly contained in the class VNP (polynomials that can be written as Hamilton cycle polynomials ${ }^{1}$ of polynomially large matrices of linear polynomials, see Section 2.1). In 2001 Mulmuley and Sohoni [31] in their Geometric Complexity Theory approach towards resolving Valiant's conjecture stated a strengthening of the conjecture (VNP $\nsubseteq \overline{\mathrm{VBP}}$ ) that is based on border complexity, which was stated independently for circuits

2021-Dec-01

1 in characteristic $\neq 2$ the Hamilton cycle polynomial can be replaced by the permanent polynomial for all our statements. 


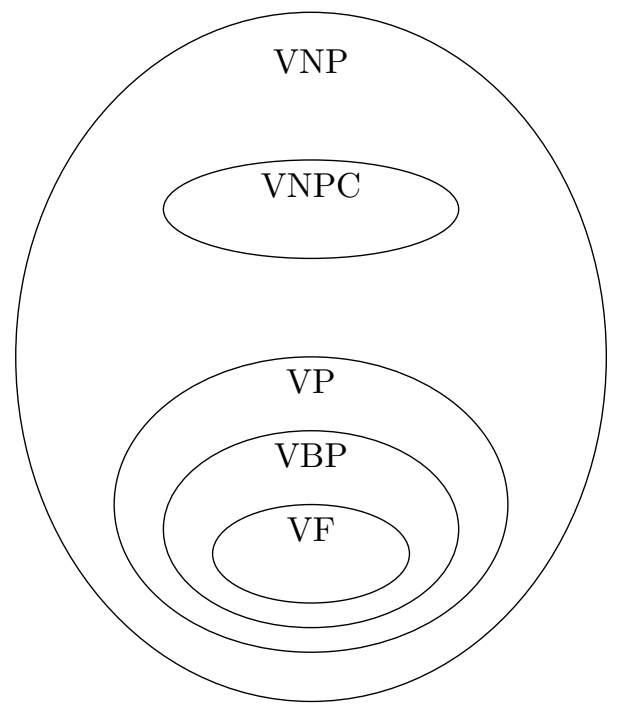

Figure 1 The known inclusions of the classical algebraic complexity classes. VF is the class of families of polynomials with polynomially sized formulas, VBP is the class of families of polynomials that can be written as polynomially large determinants of matrices of linear polynomials, VP is the class of families of polynomials with polynomially sized circuits. VNPC is the set of VNP-complete families. From a topological perspective such a depiction can be misleading, because VNPC lies dense in VNP and also VNP $\backslash$ VNPC lies dense in VNP (under p-projections), see Theorem 1.

by Bürgisser [9, hypothesis (12)] (VNP $\nsubseteq \overline{\mathrm{VP}}$ ). Border complexity was first studied in the area of fast matrix multiplication algorithms, where it led to significant algorithmic speedups $[4,3,34,32,11,37,12]$. The advantage of working with the closures of complexity classes is that this makes a large set of tools from algebraic geometry and representation theory available, see e.g. [5]. The hope is that VBP and VNP can still be separated in this coarser setting. Indeed, it is a major open question in geometric complexity theory whether or not $\mathrm{VBP}=\overline{\mathrm{VBP}}$, see [15]. If $\mathrm{VBP}=\overline{\mathrm{VBP}}$, then Valiant's conjecture must in principle be provable by algebraic geometry, provided it is true. If $\mathrm{VNP} \subseteq \overline{\mathrm{VBP}}$, then the Geometric Complexity Theory approach fails unsalvageably, while Valiant's conjecture could still be true.

In Boolean complexity theory the relationship between complexity classes is often depicted in diagrams. An analogue for the classical algebraic complexity classes is given in Figure 1. In this paper we see that such a depiction presents misleading topological information: We study the set of VNP-complete polynomials and its complement and see that surprisingly both lie dense in VNP, see Theorem 1. We prove an analogous result for VF, VBP, and VP. This highlights that the topology is very coarse.

We take the methods for proving Theorem 1 as a basis for studying VNP-completeness under different reduction notions. In particular we study border-p-projections, which were recently introduced in [7] with a focus on the border-p-projections of the iterated $2 \times 2$ matrix multiplication polynomial. We get several separations of the power of different reduction notions in Theorem 2. Our paper gives the first analysis of border reduction notions and their relative complexity in comparison to non-border reduction notions. Moreover, our paper is the first to study border complexity analogues of the so-called oracle reductions (or c-reductions) that were introduced in [8]. 


\section{Preliminaries}

\subsection{Algebraic Complexity Theory}

Fix a field $\mathbb{F}$. An algebraic circuit is defined as a rooted ${ }^{2}$ directed acyclic graph which has its leaf nodes ${ }^{3}$ labelled with variables $\left\{x_{1}, x_{2}, \ldots, x_{n}\right\}$ or field constants, and the internal nodes labelled with $\times$ ("multiplication gates") and + ("addition gates"). By induction over the circuit structure, each internal node computes a polynomial $f \in \mathbb{F}\left[x_{1}, x_{2}, \ldots, x_{n}\right]$. The output of a circuit is defined as the polynomial computed at its root. The size of an algebraic circuit is defined as the number of nodes in the circuit.

A sequence of natural numbers $(t)=\left(t_{n}\right)_{n \in \mathbb{N}}$ is called polynomially bounded if there exists a polynomial function $p$ such that for all $n \in \mathbb{N}$ we have $t_{n} \leq p(n)$. A sequence $(f)=\left(f_{n}\right)_{n \in \mathbb{N}}$ of multivariate polynomials is defined to be a p-family if the number of variables in $f_{n}$ and the degree of $f_{n}$ are both polynomially bounded. The complexity class VP is defined as the set of all p-families that have algebraic circuits whose size is polynomially bounded. If we only allow skew circuits, i.e., circuits for which each multiplication gate is adjacent to a leaf node, then we get the complexity class VBP. If instead we insist on the circuits to be rooted trees, then we get the complexity class VF. We have $\mathrm{VF} \subseteq \mathrm{VBP} \subseteq \mathrm{VP}$. See [38] or [33] for an exposition about these classes, their different definitions, and their containment.

For fixed natural numbers $N$ and $M$, a polynomial $f \in \mathbb{F}\left[x_{1}, x_{2}, \ldots, x_{N}\right]$ is said to be a projection of another polynomial $g \in \mathbb{F}\left[y_{1}, y_{2}, \ldots, y_{M}\right]$ if $f=g\left(\alpha_{1}, \alpha_{2}, \ldots, \alpha_{M}\right)$, where $\alpha_{i} \in\left\{x_{1}, x_{2}, \ldots, x_{N}\right\} \cup \mathbb{F}$. This is denoted by $f \leq g$. A p-family $(f)$ is said to be the $\mathrm{p}$ projection of another p-family $(g)$, denoted by $(f) \leq_{\mathrm{p}}(g)$, if there is a polynomially bounded function $t: \mathbb{N} \rightarrow \mathbb{N}$ such that $f_{n} \leq g_{t(n)}$ for all $n^{4}$.

p-projections were introduced by Valiant. Another natural example of reductions are c-reductions, an algebraic analogue of oracle complexity, which were introduced by Bürgisser [8]. The oracle complexity $L^{g}(f)$ of a polynomial $f$ with oracle $g$ is defined as the minimum size of a circuit with,$+ \times$, and $g$-oracle gates (the output at these gates is the computation of the polynomial $g$ on the input values. The arity of a $g$-oracle gate equals the number of variables in $g$.) that computes the polynomial $f$. Consider two p-families $(f)$ and $(g)$. The p-family $(f)$ is said to be a c-reduction of $(g)$, denoted by $(f) \leq_{\mathrm{c}}(g)$, if there exists a polynomially bounded function $t: \mathbb{N} \rightarrow \mathbb{N}$ such that $L^{g_{t(n)}}\left(f_{n}\right)$ is polynomially bounded. It is clear that $(f) \leq_{\mathrm{p}}(g)$ implies $(f) \leq_{\mathrm{c}}(g)$.

Let $\mathfrak{S}_{n}$ denote the symmetric group on $n$ symbols. The determinant is the sequence of homogeneous degree $n$ polynomials in $n^{2}$ many variables defined via $\operatorname{det}_{n}:=$ $\sum_{\pi \in \mathfrak{S}_{n}} \operatorname{sign}(\pi) \prod_{i=1}^{n} x_{i, \pi(i)}$. Valiant showed that $(f) \in \mathrm{VBP}$ if and only if $(f) \leq_{\mathrm{p}}$ (det).

Let $C_{n} \subseteq \mathfrak{S}_{n}$ be the subset of length $n$ cycles. The Hamiltonian cycle family (HC) is the sequence of homogeneous degree $n$ polynomials $\mathrm{HC}_{n}$ on $n^{2}$ many variables defined via $\mathrm{HC}_{n}:=\sum_{\pi \in C_{n}} \prod_{i=1}^{n} x_{i, \pi(i)}$. We define the class VNP as the set of all p-families $(f)$ that satisfy $(f) \leq_{\mathrm{p}}(\mathrm{HC})$. This is known to be equivalent to $(f) \leq_{\mathrm{c}}(\mathrm{HC})$. Often a different definition is given that is closer in spirit to the counting complexity class \#P, where VNP is defined as a summation of a VP function over the Boolean hypercube, but Valiant [39] showed that these definitions are equivalent. In particular, it is easy to see that $\mathrm{VP} \subseteq \mathrm{VNP}$. Valiant's famous conjectures are VF $\neq \mathrm{VNP}, \mathrm{VBP} \neq \mathrm{VNP}$, and VP $\neq \mathrm{VNP}$. To find

2 The digraph has a single node with out-degree 0.

3 Leaf nodes are the nodes with in-degree 0.

4 Note the non-uniformity of this notion of reduction as we get to choose the constants $a l p h a_{i}$ in the projection fresh (without complexity constraints) for every $n$. 
candidates of $\mathrm{p}$-families in VNP that are outside of VF, VBP or VP, the following notion of VNP-completeness is useful.

A p-family $(f)$ is defined to be VNP-p-complete if $(f) \in \mathrm{VNP}$ and $(\mathrm{HC}) \leq_{\mathrm{p}}(f)$. The set of all VNP-p-complete $\mathrm{p}$-families is denoted by $\operatorname{VNPC}\left(\leq_{\mathrm{p}}\right)$. Analogously, a p-family $(f)$ is defined to be VNP-c-complete if $(f) \in \mathrm{VNP}$ and $(\mathrm{HC}) \leq_{\mathrm{c}}(f)$. The set of all VNP-c-complete p-families is denoted by $\operatorname{VNPC}\left(\leq_{c}\right)$. It is clear that $\operatorname{VNPC}\left(\leq_{p}\right) \subseteq \operatorname{VNPC}\left(\leq_{c}\right)$. The fact that these two sets are different was established in [20] with a short argument.

The main motivation behind VNP-completeness comes from the following simple observation: If we would find $(g)$ such that both $(g) \in \operatorname{VNPC}\left(\leq_{\mathrm{p}}\right)$ and $(g) \in \mathrm{VP}$, then for all $(f) \in \mathrm{VNP}$ we would have $(f) \leq_{\mathrm{p}}(\mathrm{HC}) \leq_{\mathrm{p}}(g)$, and by transitivity $(f) \leq_{\mathrm{p}}(g)$, which implies $(f) \in \mathrm{VP}$, thus VP $=$ VNP. This observation similarly holds for VF and VBP instead of VP.

As the natural variation of the above, a p-family $(f)$ is defined to be VF-p-complete if $(f) \in \mathrm{VF}$ and $(g) \leq_{\mathrm{p}}(f)$ for every $(g) \in \mathrm{VF}$. The set of all VF-p-complete $\mathrm{p}$-families is denoted by $\operatorname{VFC}\left(\leq_{\mathrm{p}}\right)$. The iterated $3 \times 3$ matrix multiplication polynomial family is an example of an element in $\operatorname{VFC}\left(\leq_{\mathrm{p}}\right)$ [2]. A p-family $(f)$ is defined to be VBP-p-complete if $(f) \in \mathrm{VBP}$ and $(g) \leq_{\mathrm{p}}(f)$ for every $(g) \in \mathrm{VBP}$. The set of all VBP-p-complete $\mathrm{p}$-families is denoted by $\operatorname{VBPC}\left(\leq_{\mathrm{p}}\right)$. The determinant polynomial family is an example of an element in $\operatorname{VBPC}\left(\leq_{\mathrm{p}}\right)$, see [38]. A p-family $(f)$ is defined to be VP-p-complete if $(f) \in \mathrm{VP}$ and $(g) \leq_{\mathrm{p}}(f)$ for every $(g) \in \mathrm{VP}$. The set of all VP-p-complete $\mathrm{p}$-families is denoted by $\operatorname{VPC}\left(\leq_{\mathrm{p}}\right)$. There exist specific graph homomorphism polynomial families that are in $\operatorname{VPC}\left(\leq_{p}\right)$, see the recent [29]. This resolved a long-standing open problem of finding natural problems in $\operatorname{VPC}\left(\leq_{p}\right)$.

\subsection{Border complexity and topology}

In this section we define so-called border complexity analogues to the reduction notions in Section 2.1. This was first done explicitly in [7]. In the rest of the paper we assume that our base field $\mathbb{F}$ is $\mathbb{Q}, \mathbb{R}$, or $\mathbb{C}^{5}$. The ring $P$ of polynomials over $\mathbb{F}$ in any number of variables is a countably infinite dimensional metric vector space with the coefficient-wise Euclidean metric $\rho(f, g):=\|f-g\|$, where $\|$.$\| is the Euclidean norm of the vector of coefficients. We define$ $R$ to be the vector space of sequences $(f):=\left(f_{n}\right)_{n \in \mathbb{N}}$ of elements from $P$ :

$$
R:=P^{\mathbb{N}} .
$$

Let

$$
D:=\left(\mathbb{R}_{>0}\right)^{\mathbb{N}}
$$

be the set of sequences of positive real numbers. We endow $R$ with the box topology ${ }^{6}$ : The basic open sets are the sequences of open balls of radii $(\delta) \in D$ around elements $(f) \in R$,

5 This also means that the Hamilton cycle polynomial can be replaced by the permanent polynomial for all our statements [39]. We chose HC to be consistent with [20], which also studies other fields. In Lemma 5 we require the field to be large enough. In Lemma 7 we require the field to be a subfield of $\mathbb{C}$, but it is possible to phrase our density results in a field-independent way that is purely algebraic, see [22]. We focus only on the most natural case of defining closures, which is over an infinite field such that we have a metric on $P$.

${ }^{6}$ The usual product topology used in an early preprint of this paper [21] is too coarse to correctly capture the definitions of $\overline{\mathrm{VBP}}$ etc from the literature, which was kindly pointed out by Josh Grochow. On the other hand, the closure in [7] is only defined for subsets of $R$ that are defined via an upper bound on an algebraic complexity measure, which provides no way to take the closure of the set of VNP-complete p-families, or of their complement. Since we are the first to define a closure operator that works for all subsets of $R$, in Section 7 we prove that our definition coincides with the defintion from the literature in all cases. 
formally

$$
B_{(f),(\delta)}:=\left\{(g) \in R \mid \forall n \in \mathbb{N}: \rho\left(f_{n}, g_{n}\right)<\delta_{n}\right\} .
$$

A subset of $R$ is called open in $R$ if it is the (possibly infinite) union of basic open sets. A subset $A$ of $R$ is called closed in $R$ if $R \backslash A$ is open in $R$. The closure $\bar{A}$ of $A$ in $R$ is the smallest closed subset of $R$ containing $A^{7}$. Since VNP $\subseteq R$, we have that VF, VBP, VP, and VNP are topological spaces using the subspace topology ${ }^{8}$.

In so-called sequential topological spaces the closure $\bar{A}$ can be described as the set of all limit points of sequences in $A$. But $R$ is not a sequential space (and hence not first-countable). So we have to use the more general notion of convergence of nets instead of sequences. We now recall the standard definition of net convergence and give a natural example in our setting. A downward-directed set $\mathcal{D}$ is a nonempty set with a reflexive and transitive binary relation $\preceq$ (i.e., a preorder) with the additional property that for all $\varepsilon^{\prime} \in \mathcal{D}$ and $\varepsilon^{\prime \prime} \in \mathcal{D}$ there exists $\varepsilon^{\prime \prime \prime} \in \mathcal{D}$ with $\varepsilon^{\prime \prime \prime} \preceq \varepsilon^{\prime}$ and $\varepsilon^{\prime \prime \prime} \preceq \varepsilon^{\prime \prime} .{ }^{9}$ For a topological space $X$ a net $((g))$ in $X$ is a function $\mathcal{D} \rightarrow X$, where $\mathcal{D}$ is a downward-directed set. We write $\left(g_{\varepsilon}\right)$ for its evaluation at $\varepsilon \in \mathcal{D}$. We say a net $((g))$ converges to a point $(f) \in X$ if for every open set $U$ containing $(f)$ there exists $\varepsilon \in \mathcal{D}$ such that for all $\varepsilon^{\prime} \preceq \varepsilon$ we have $\left(g_{\varepsilon^{\prime}}\right) \in U$. Note that it is equivalent to require this only for basic open sets $U$. If $((g))$ converges to $(f)$, then we call $(f)$ a limit point of $((g))$. The key property of nets is that for every topological space $X$ and every subset $A \subseteq X$ we have that $(f) \in \bar{A}$ if and only if there exists a net in $A$ that converges to $(f)$, see e.g. [24, Ch. 2, 2 Theorem (b)]. ${ }^{10}$

- Example $(*)$. For an illustrative example on how nets are used, fix the ground field to be $\mathbb{C}$ and recall that the Waring rank of a homogeneous degree $n$ polynomial $f$ is defined as the smallest number $r$ such that homogeneous linear polynomials $\ell_{i}$ exist, $1 \leq i \leq r$, with $f=\sum_{i=1}^{r} \ell_{i}^{n}$. For example, the Waring rank of $x_{1}^{2} x_{2}$ is at most 3 , because $6 x_{1}^{2} x_{2}=$ $\left(x_{1}+x_{2}\right)^{3}-\left(x_{1}-x_{2}\right)^{3}-\left(x_{2}\right)^{3}$. Let VWaring 2 denote the set of all sequences of $\mathrm{p}$-families of homogeneous polynomials of Waring rank at most 2. Let $(f) \in R$ be the p-family of homogeneous degree $n$ polynomials defined via $f_{n}:=x_{1}^{n-1} x_{2}$. Recall the downward-directed set $D:=\left(\mathbb{R}_{>0}\right)^{\mathbb{N}}$. For every $n \in \mathbb{N},(\varepsilon) \in D$, we define $g_{n,(\varepsilon)}:=\frac{1}{n \varepsilon_{n}}\left(x_{1}+\varepsilon_{n} x_{2}\right)^{n}-\frac{1}{n \varepsilon_{n}} x_{1}^{n}$. Clearly, for every fixed $(\varepsilon) \in D$ we have $\left(g_{(\varepsilon)}\right):=\left(g_{n,(\varepsilon)}\right)_{n \in \mathbb{N}} \in$ VWaring $_{2}$, so $((g))$ is a net in VWaring $_{2}$. To show that $(f) \in \overline{\text { VWaring }}_{2}$ it remains to show that the net $((g))$ converges to $(f)$. This can be seen as follows. For every $(\delta) \in D$ we can choose sufficiently small $(\varepsilon) \in D$ such that $\left(g_{(\varepsilon)}\right) \in B_{(f),(\delta)}$. Since for all $\varepsilon^{\prime} \preceq \varepsilon$ and all $n$ we have $\rho\left(g_{n,\left(\varepsilon^{\prime}\right)}, f_{n}\right) \leq \rho\left(g_{n,(\varepsilon)}, f_{n}\right)$, it follows that for all $\varepsilon^{\prime} \preceq \varepsilon$ we have $\left(g_{\left(\varepsilon^{\prime}\right)}\right) \in B_{(f),(\delta)}$. Hence $((g))$ converges to $(f)$. In the rest of this paper we will always use this simple form of the convergence of nets.

A subset $A \subseteq X$ is defined to be dense in $X$ if $\bar{A}=X$, i.e., every point in $X$ is a limit of a converging net of elements in $A$. For all the sets VF, VBP, VP, VNP, it is a major open

\footnotetext{
7 The closure notation with the overline was first introduced in [10]. The earlier work [9] used an underline, which is natural when thinking in terms of polynomially bounded border complexity measures.

8 The box topology construction also works with other topologies on $P$, so that in principle it can be used over finite fields as well.

${ }^{9}$ One important example that we will use often is the set of sequences of positive real numbers $\mathcal{D}=D$ with elementwise comparison. Usually in the definition of nets the sets are upward-directed instead of downward-directed, but downward-directed sets fit better with our intuition of $\varepsilon$ getting smaller and smaller.

${ }^{10}$ Note that if we change the definition of downward-directed sets to upward-directed and let $\mathcal{D}$ be $\mathbb{N}$, then we recover the usual definition of sequences.
} 
question if they are equal to their closure, see [15]. In this paper we restrict our attention to the subspace topology on $\mathrm{VNP} \subseteq R$.

The notion of border projections is closely related to the concept of closures, but it does not require the use of nets. A polynomial $f$ is called a border projection of a polynomial $g$, if there exists a sequence $\left(g_{1}, g_{2}, \ldots\right)$ with $g_{i} \leq g$ for all $i$ and $\lim _{m \rightarrow \infty} g_{m}=f$. Then we write $f \unlhd g$. Clearly $f \leq g$ implies $f \unlhd g$. We extend this definition to sequences of polynomials as follows. A sequence of polynomials $(f)$ is defined to be a border p-projection of another sequence of polynomials $(g)$, denoted by $(f) \unlhd_{p}(g)$, if there is a polynomially bounded function $t: \mathbb{N} \rightarrow \mathbb{N}$ such that $f_{n} \unlhd g_{t(n)}$ for all $n$. ${ }^{11}$

The border oracle complexity $\overline{L^{g}}(f)$ of a polynomial $f$ with oracle access to $g$ is defined as the smallest $r$ such that a sequence of polynomials $\left(g_{1}, g_{2}, \ldots\right)$ exists with $L^{g}\left(g_{m}\right) \leq r$ for all $m$ and $\lim _{m \rightarrow \infty} g_{m}=f$. Clearly $\overline{L^{g}}(f) \leq L^{g}(f)$ for all $f$ and $g$. Consider two p-families of polynomials, $(f)$ and $(g)$. The p-family $(f)$ is said to be a border c-reduction of $(g)$, denoted by $(f) \unlhd_{c}(g)$, if there exists a polynomially bounded function $t: \mathbb{N} \rightarrow \mathbb{N}$ such that $\overline{L^{g_{t(n)}}}\left(f_{n}\right)$ is polynomially bounded.

A p-family $(f)$ is defined to be VNP-border-p-complete if $(f) \in \mathrm{VNP}$ and $(\mathrm{HC}) \unlhd_{\mathrm{p}}(f)$. The set of all VNP-border-p-complete $\mathrm{p}$-families is denoted by $\operatorname{VNPC}\left(\unlhd_{\mathrm{p}}\right)$. Analogously, a p-family $(f)$ is defined to be VNP-border-c-complete if $(f) \in \mathrm{VNP}$ and $(\mathrm{HC}) \unlhd_{c}(f)$. The set of all VNP-border-c-complete p-families is denoted by $\operatorname{VNPC}\left(\unlhd_{c}\right)$.

In geometric complexity theory the commonly used type of reduction is similar to pprojections and border-p-projections, but instead of replacing variables with constants and variables, variables are replaced by affine linear polynomials [10]. All proofs in this paper also work with this version of p-projections and border-p-projections.

\section{Main results}

Using a fairly elementary proof we obtain the following surprising density results.

- Theorem 1. Let $\leq$ be any of $\leq_{p}, \leq_{c}, \unlhd_{p}$, or $\unlhd_{c}$. Let $\lesssim$ be any of $\leq_{p}, \unlhd_{p}$. Then:

- the set $\mathrm{VNPC}(\leq)$ is a dense subset of $\mathrm{VNP}$ and the complement $\mathrm{VNP} \backslash \mathrm{VNPC}(\lesssim)$ is a dense subset of VNP.

Analogously,

- $\mathrm{VFC}(\leq)$ and $\mathrm{VF} \backslash \mathrm{VFC}(\lesssim)$ are both dense in $\mathrm{VF}$,

- $\operatorname{VBPC}(\leq)$ and $\mathrm{VBP} \backslash \operatorname{VBPC}(\lesssim)$ are both dense in $\mathrm{VBP}$,

- $\operatorname{VPC}(\leq)$ and $\mathrm{VP} \backslash \mathrm{VPC}(\lesssim)$ are both dense in $\mathrm{VP}$.

The multitude of density statements in Theorem 1 suggests that the topology on $R$ is suprisingly coarse (i.e., has few open sets). We leave it as an open problem if $\operatorname{VNP} \backslash \operatorname{VNPC}(\leq)$ is dense in VNP for the other two reduction notions $\leq_{c}$ and $\unlhd_{c}$. Oracle complexity is too coarse to study VF-completeness, VBP-completeness, or VP-completeness, because a polynomially sized circuit does not gain any benefit from having oracle access to a polynomially sized formula or a polynomially sized determinant, or another polynomially sized circuit.

As a second result we initiate the comparison of classical and border reduction notions. We give an almost complete separation of the sets of VNP-complete polynomials under the different reduction notions as follows.

\footnotetext{
${ }^{11}$ We remark that in the literature the class $\overline{\mathrm{VBP}}$ is usually defined via: $(f) \in \overline{\mathrm{VBP}}$ if and only if $(f) \unlhd_{p}$ (det). We prove in Section 7 that this coincides with our definition of $\overline{\mathrm{VBP}}$. Analogously for VF, VP, VNP and their respective complete polynomials.
} 
- Theorem 2. We have the following diagram of inclusions (solid arrows) and non-inclusions (dashed arrows).

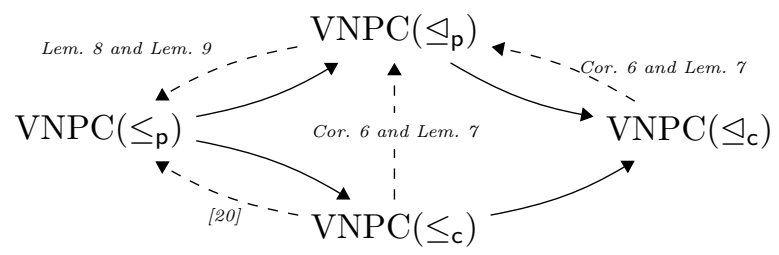

Analogously for VF, VBP, and VP instead of VNP.

The inclusions (solid arrows) are obvious consequences of the relations among $\leq_{\mathrm{p}}$, $\leq_{\mathrm{c}}$ etc. The non-inclusions are proved in the respective lemmas as annotated in the figure. We only treat the case VNP, as the other classes are handled in exactly the same way, with replacing (HC) by the respective complete p-family. One caveat: Lemma 5 does not work out of the box for VF. We treat this case in Section 6.1.

\section{$4 \quad$ Related work}

The relative power of reductions in the Boolean world has been analyzed for example in $[17,18]$. The notion of c-reductions in algebraic complexity theory is relatively new [8]. The difference between p-projections and c-reductions plays a prominent role in [29]. The relative power of algebraic reduction notions has been studied before: [20] show with a short argument that

$\operatorname{VNPC}\left(\leq_{\mathrm{p}}\right) \varsubsetneqq \operatorname{VNPC}\left(\leq_{\mathrm{c}}\right)$.

They do not study border complexity though.

Border complexity has already been an object of study in algebraic complexity theory for bilinear maps since 1980 (see [3]) and is still a very active area of research today [27, 26]. The study of border complexity for polynomials has recently gained significant momentum, see for example $[15,25,7,6]$. In fact, [7] prove that $\operatorname{VFC}\left(\leq_{\mathrm{p}}\right) \varsubsetneqq \operatorname{VFC}\left(\unlhd_{\mathrm{p}}\right)$. This result makes use of a result on width- 2 ABPs that is obtained from a fairly involved analysis [1].

\section{Proof of Theorem 1}

We start with an observation of [20] that we state simultaneously for VNP, VF, VBP, and VP. It is clear that the result holds in much higher generality.

- Proposition 3. Suppose $(f) \in \mathrm{VNP}[\mathrm{VF}, \mathrm{VBP}, \mathrm{VP}]$ and each $f_{n}$ has the following form: $f_{n}=q\left(r g_{n}+c_{n} g_{n}^{2}\right)$ for some $\mathrm{p}$-family $(g)$, some fixed polynomial $r$ of any degree $d_{2}$, some fixed polynomial $q$ of even degree $d_{1}$ that is also a perfect square, and some sequence $(c)$ of nonzero constants. Then $(f) \notin \operatorname{VNPC}\left(\leq_{\mathrm{p}}\right)\left[\operatorname{VFC}\left(\leq_{\mathrm{p}}\right), \operatorname{VBPC}\left(\leq_{\mathrm{p}}\right), \operatorname{VPC}\left(\leq_{\mathrm{p}}\right)\right]$.

Proof. The proof is a minor generalization of [20, Lemma 3.2]. Consider a univariate polynomial $s(y)$ of odd degree $M>d_{1}+2 d_{2}$. We claim that $s$ cannot be written as a projection of $f_{n}$, for any $n$. Let $\operatorname{deg}_{y}(h)$ denote the degree of a polynomial $h$ in the variable $y$, when considered as a polynomial over the polynomial ring in all its other constituent variables. Let $\gamma$ be any linear projection map in the sense of $\leq_{\mathrm{p}}$. Then, $\operatorname{deg}_{y}\left(\gamma\left(f_{n}\right)\right) \leq \max \left[\operatorname{deg}_{y}\left(\gamma\left(q \cdot r \cdot g_{n}\right)\right), \operatorname{deg}_{y}\left(\gamma\left(q \cdot g_{n}^{2}\right)\right)\right]$. Also, note that $\operatorname{deg}_{y}(\gamma(q)) \leq d_{1}$ and $\operatorname{deg}_{y}(\gamma(r)) \leq d_{2}$. 
If $\operatorname{deg}_{y}\left(\gamma\left(g_{n}\right)\right) \leq d_{2}$, then $\operatorname{deg}_{y}\left(\gamma\left(f_{n}\right)\right) \leq d_{1}+2 d_{2}$. If $\gamma\left(f_{n}\right)=s(y)$, this contradicts the fact that $s(y)$ has degree $M$.

Otherwise, $\operatorname{deg}_{y}\left(\gamma\left(g_{n}\right)\right)>d_{2}$ and hence $\operatorname{deg}_{y}\left(\gamma\left(f_{n}\right)\right)=\operatorname{deg}_{y}(\gamma(q))+$ $\max \left\{\operatorname{deg}_{y}\left(\gamma\left(r g_{n}\right)\right), \operatorname{deg}_{y}\left(\gamma\left(c_{n} g_{n}^{2}\right)\right)\right\}=\operatorname{deg}_{y}\left(\gamma\left(q \cdot g_{n}^{2}\right)\right)$, because $\operatorname{deg}_{y}(\gamma(r)) \leq d_{2}$. But $q \cdot g_{n}^{2}$ is a perfect square polynomial, hence $\operatorname{deg}_{y}\left(\gamma\left(f_{n}\right)\right)$ is even, but $s(y)$ has odd degree. Hence, $\gamma\left(f_{n}\right) \neq s(y)$.

Thus, the constant sequence $(s)$ cannot be written as a p-projection of $f$. Hence, $(f) \notin \operatorname{VNPC}\left(\leq_{\mathrm{p}}\right)\left[\operatorname{VFC}\left(\leq_{\mathrm{p}}\right), \operatorname{VBPC}\left(\leq_{\mathrm{p}}\right), \operatorname{VPC}\left(\leq_{\mathrm{p}}\right)\right]$

In a simpler setting the above ideas work also for border reductions, as the following proposition shows.

- Proposition 4 (border reduction version of Prop. 3). Let $g$ be a non-constant polynomial with $g \unlhd f+c f^{2}$ for a nonzero field constant $c$. Then $\operatorname{deg}(g)$ is even.

Proof. A classical theorem by Lehmkuhl and Lickteig [28] over $\mathbb{F} \in\{\mathbb{C}, \mathbb{R}\}$ (published for order 3 tensors, but frequently its generalization to arbitrary polynomials is used, see e.g. $[9,14,16,35,19,30])$ implies that the approximation of $f$ can be written in terms of Laurent polynomials: $f^{\prime}:=\left(f+c f^{2}\right)(\bar{\alpha})$,

$$
\lim _{\varepsilon \rightarrow 0}\left(f^{\prime}\left(\alpha_{1}, \alpha_{2}, \ldots, \alpha_{N}\right)\right)=g
$$

for some $\alpha_{i} \in \mathbb{F}\left[\varepsilon, \varepsilon^{-1}\right] \cup \overline{\mathbf{x}}$. If all homogeneous components of $f(\bar{\alpha})$ converge for $\varepsilon \rightarrow 0$, then $\lim _{\varepsilon \rightarrow 0}\left(f^{\prime}\right)=\lim _{\varepsilon \rightarrow 0} f(\bar{\alpha})+c\left(\lim _{\varepsilon \rightarrow 0} f(\bar{\alpha})\right)^{2}$. So the top homogeneous part of $\lim _{\varepsilon \rightarrow 0}\left(f^{\prime}\right)$ is (up to rescaling with $c$ ) the square of the top homogeneous part of $\lim _{\varepsilon \rightarrow 0}(f(\bar{\alpha})$ ). Hence in this case the degree is even.

We now treat the case where there exists a homogeneous component of $f(\bar{\alpha})$ that diverges for $\varepsilon \rightarrow 0$. Let $d$ be the largest degree of such a component. Assume for now that $d \neq 0$. Then, the homogeneous degree $2 d$ component of $f^{2}(\bar{\alpha})$ must diverge and this cannot be cancelled out by the other homogeneous components which are of lower degree. This is a contradiction to the convergence of the sum. Hence, $d=0$.

Now assume that the homogeneous degree 0 component $f(\bar{\alpha})_{0}$ of $f(\bar{\alpha})$ diverges for $\varepsilon \rightarrow 0$ and let $M<0$ be the smallest exponent of $\varepsilon$ in the univariate Laurent polynomial $f(\bar{\alpha})_{0}$. This implies that the homogeneous degree 0 component of $f^{\prime}$ is a Laurent polynomial in $\varepsilon$ that has a nonzero term of degree $2 M$. Hence $f^{\prime}$ diverges, which is a contradiction to our initial assumption that $\lim _{\varepsilon \rightarrow 0} f^{\prime}=g$.

Proof of Theorem 1. First we prove that $\operatorname{VNPC}\left(\leq_{p}\right)$ is dense in VNP. Let $(f) \in$ VNP be arbitrary. Recall the downward directed set $D:=\left(\mathbb{R}_{>0}\right)^{\mathbb{N}}$. For every $n \in \mathbb{N},(\varepsilon) \in D$, we define

$$
g_{n,(\varepsilon)}:=f_{n}+\varepsilon_{n} y_{n}\left(\mathrm{HC}_{n}-f_{n}\right),
$$

where each $y_{n}$ is a variable that is unused by $f_{n}$ and $\mathrm{HC}_{n}$ for all $n$. For every fixed $(\varepsilon) \in D$ we have $\left(g_{(\varepsilon)}\right) \in \mathrm{VNP}$, because VNP is closed under taking finitely many sums and products. Moreover, we have $\left(g_{(\varepsilon)}\right) \in \operatorname{VNPC}\left(\leq_{\mathrm{p}}\right)$, which can be seen as follows. Indeed, $(\mathrm{HC}) \leq_{\mathrm{p}}\left(g_{(\varepsilon)}\right)$, because under the projection map $\gamma: y_{n} \mapsto \frac{1}{\varepsilon_{n}}$ we have $\gamma\left(g_{n,(\varepsilon)}\right)=\mathrm{HC}_{n}$ and thus $\gamma\left(\left(g_{(\varepsilon)}\right)\right)=(\mathrm{HC})$. Hence $((g))$ is a net in $\operatorname{VNPC}\left(\leq_{\mathrm{p}}\right)$.

The net $((g))$ converges to $(f)$, which can be seen in the same way as in Example $(*)$ : For every $(\delta) \in D$ we can choose sufficiently small $(\varepsilon) \in D$ such that $\left(g_{(\varepsilon)}\right) \in B_{(f),(\delta)}$. Since 
for all $\varepsilon^{\prime} \preceq \varepsilon$ and all $n$ we have $\rho\left(g_{n,\left(\varepsilon^{\prime}\right)}, f_{n}\right) \leq \rho\left(g_{n,(\varepsilon)}, f_{n}\right)$, it follows that for all $\varepsilon^{\prime} \preceq \varepsilon$ we have $\left(g_{\left(\varepsilon^{\prime}\right)}\right) \in B_{(f),(\delta)}$.

The result for the other reduction types is immediate, because p-projections are the weakest notion of reduction we consider, in particular $\operatorname{VNPC}\left(\leq_{\mathrm{p}}\right) \subseteq \operatorname{VNPC}\left(\leq_{\mathrm{c}}\right), \operatorname{VNPC}\left(\leq_{\mathrm{p}}\right) \subseteq$ $\operatorname{VNPC}\left(\unlhd_{p}\right)$, and $\operatorname{VNPC}\left(\leq_{p}\right) \subseteq \operatorname{VNPC}\left(\unlhd_{c}\right)$.

For the other part, let $(f) \in \mathrm{VNP}$ be arbitrary. For every $n \in \mathbb{N},(\varepsilon) \in D$, we define

$$
h_{n,(\varepsilon)}:=f_{n}+\varepsilon_{n} f_{n}^{2}
$$

Obviously $\left(h_{(\varepsilon)}\right) \in \mathrm{VNP}$, but according to Proposition 4 we have that the constant p-family of the degree 1 polynomial $(x)$ has $(x) \unlhd_{\mathbf{p}}\left(h_{(\varepsilon)}\right)$ and hence $\left(h_{(\varepsilon)}\right) \notin \operatorname{VNPC}\left(\unlhd_{\mathbf{p}}\right)$. Hence $((h))$ is a net in $\operatorname{VNP} \backslash \operatorname{VNPC}\left(\unlhd_{\mathrm{p}}\right)$. Clearly $((h))$ converges to $(f)$ with the same argument as for $((g))$ in the first part of this proof. Thus, $\operatorname{VNP} \backslash \operatorname{VNPC}\left(\unlhd_{p}\right)$ is dense in VNP.

The analogous statements about VF, VBP, and VP that are claimed in the theorem are proved in exactly the same way by replacing $\mathrm{HC}_{n}$ by a complete polynomial family for the respective class.

\section{Proof of Theorem 2}

We start with a classical lemma about taking roots.

- Lemma 5. Suppose $g=f^{r}$ for some $f \in \mathbb{F}[\overline{\mathbf{x}}]$ of degree $d$ and constant $r$, where $\overline{\mathbf{x}}$ denotes a set of variables. Then $f$ can be computed by a g-oracle circuit of size poly $(d)$. In particular, if $g_{n}=f_{n}^{r}$ for all $n$, then $(f) \leq_{c}(g)$.

Proof. (The proof follows that of a special case of [23], the proof technique borrows from [40] and [13]). Consider a polynomial $g=f^{r}$, where $f$ has degree $d$. Notice that for every infinite field $\mathbb{F}$ and every nonzero polynomial $f$ over $\mathbb{F}[\overline{\mathbf{x}}]$, there exists $\bar{\alpha} \in \mathbb{F}|\overline{\mathbf{x}}|$, such that $f(\bar{\alpha}) \neq 0$. Also, shifting the variables $f(\overline{\mathbf{x}}) \mapsto f(\overline{\mathbf{x}}+\alpha)$ is an invertible operation since you may re-shift at the input nodes of the circuit. Thus, by appropriately shifting we may assume w.l.o.g. that $g(\mathbf{0}) \neq 0$. Rescaling at the output node is also an invertible operation, so we may assume w.l.o.g. that $g(\mathbf{0})=1$. We can write:

$$
f=(1+(g-1))^{1 / r} .
$$

Using the binomial theorem for rational exponents, this gives us: $(1+(g-1))^{1 / r}=$

$$
1+\frac{1}{r}(g-1)+\left(\begin{array}{c}
1 / r \\
2
\end{array}\right)(g-1)^{2}+\cdots+\left(\begin{array}{c}
1 / r \\
d
\end{array}\right)(g-1)^{d}+\cdots
$$

Since $g(\mathbf{0})=1$, then $g-1=0 \bmod (\overline{\mathbf{x}})$. Thus, $(g-1)^{i}$ has only monomials of degree larger than $d$ for $i \geq d+1$. So, $f=$

$$
1+\frac{1}{r}(g-1)+\left(\begin{array}{c}
1 / r \\
2
\end{array}\right)(g-1)^{2}+\cdots+\left(\begin{array}{c}
1 / r \\
d
\end{array}\right)(g-1)^{d} \bmod \left(\left(_{\mathbf{x}}^{d+1}\right)\right.
$$

where $\overline{\mathbf{x}}^{d+1}$ denotes the set of all monomials of degree $d+1$. We have the oracle circuit for $g$. The modular operation can be done via Strassen's homogenization trick [36]. Specifically, each homogeneous part of $f$ can be written as a linear combination of $(d+1)$ p-projections of $g$. Thus, computing roots using oracle gates is possible with circuits of size poly $(d)$. This proves the first part. The second part follows from the fact that $\mathrm{p}$-families have polynomially bounded degrees. 
As an immediate corollary we obtain:

- Corollary 6. $\left(\mathrm{HC}^{2}\right) \in \operatorname{VNPC}\left(\leq_{c}\right)$.

Proof. By Lemma 5 we have $(\mathrm{HC}) \leq_{c}\left(\mathrm{HC}^{2}\right)$. Since $(\mathrm{HC}) \in \operatorname{VNPC}\left(\leq_{c}\right)$, for every $(f) \in$ VNP we have $(f) \leq_{c}(\mathrm{HC})$. By transitivity we have $(f) \leq_{c}\left(\mathrm{HC}^{2}\right)$. Therefore $\left(\mathrm{HC}^{2}\right) \in$ $\operatorname{VNPC}\left(\leq_{c}\right)$.

- Lemma 7. $\left(\mathrm{HC}^{2}\right) \notin \operatorname{VNPC}\left(\unlhd_{\mathrm{p}}\right)$.

Proof. We claim that every border-p-projection of $\mathrm{HC}_{n}^{2}$ is the square of a polynomial. The lemma follows from this fact, because for example the polynomial $x^{3}$ is not a square, but the constant p-family $\left(x^{3}\right)$ is in VNP.

Let $f \in \mathbb{F}[\overline{\mathbf{x}}]$ be a border-p-projection of $\mathrm{HC}_{n}^{2}$. We use [28] again:

$$
\lim _{\varepsilon \rightarrow 0} \operatorname{HC}_{n}^{2}\left(\alpha_{1}, \alpha_{2}, \ldots, \alpha_{n^{2}}\right)=f
$$

for some $\alpha_{i} \in \mathbb{F}\left[\varepsilon, \varepsilon^{-1}\right] \cup \overline{\mathbf{x}}$. We interpret $\operatorname{HC}_{n}\left(\alpha_{1}, \alpha_{2}, \ldots, \alpha_{n^{2}}\right)$ as a univariate Laurent polynomial in $\varepsilon$ with coefficients in $\mathbb{F}[\overline{\mathbf{x}}]$. Let $M$ be the smallest exponent of $\varepsilon$. If $M<0$, then the univariate Laurent polynomial $\mathrm{HC}_{n}^{2}\left(\alpha_{1}, \alpha_{2}, \ldots, \alpha_{n^{2}}\right)$ has a nonzero degree $2 M$ term, which means that $\operatorname{HC}_{n}^{2}\left(\alpha_{1}, \alpha_{2}, \ldots, \alpha_{n^{2}}\right)$ does not converge for $\varepsilon \rightarrow 0$. This is ruled out by (1). Therefore $M \geq 0$ and hence $L:=\lim _{\varepsilon \rightarrow 0} \operatorname{HC}_{n}\left(\alpha_{1}, \alpha_{2}, \ldots, \alpha_{n^{2}}\right)$ exists. Therefore, by continuity we have $f=\lim _{\varepsilon \rightarrow 0} \operatorname{HC}_{n}^{2}\left(\alpha_{1}, \alpha_{2}, \ldots, \alpha_{n^{2}}\right)=\left(\lim _{\varepsilon \rightarrow 0} \operatorname{HC}_{n}\left(\alpha_{1}, \alpha_{2}, \ldots, \alpha_{n^{2}}\right)\right)^{2}=$ $L^{2}$.

We now construct $(P) \in \operatorname{VNPC}\left(\unlhd_{\mathrm{p}}\right) \backslash \operatorname{VNPC}\left(\leq_{\mathrm{p}}\right)$ via

$$
P_{n}:=z^{2}\left(y \mathrm{HC}_{n}+y^{2} \mathrm{HC}_{n}^{2}\right)
$$

where $y$ and $z$ are variables outside the set of variables in $\mathrm{HC}_{n}$, for all $n$.

- Lemma 8. $(P) \notin \operatorname{VNPC}\left(\leq_{\mathrm{p}}\right)$

Proof. This is a direct consequence of Proposition 3 with $g_{n}=y \mathrm{HC}_{n}, c_{n}=r=1, q=z^{2}$.

> Lemma 9. $(P) \in \operatorname{VNPC}\left(\unlhd_{\mathrm{p}}\right)$.

Proof. Consider the projection map $\gamma_{\varepsilon}$ defined by:

$$
y \mapsto \varepsilon^{2} \quad \text { and } \quad z \mapsto \frac{1}{\varepsilon}
$$

For every $n \in \mathbb{N}$ we have $\gamma_{\varepsilon}\left(P_{n}\right) \leq P_{n}$ and $\gamma_{\varepsilon}\left(P_{n}\right)=\mathrm{HC}_{n}+\varepsilon^{2} \mathrm{HC}_{n}^{2}$. Hence $\mathrm{HC}_{n} \unlhd P_{n}$. Therefore (HC) $\unlhd_{\mathrm{p}}(P)$, which finishes the proof.

\subsection{Arithmetic formulas}

Lemma 5 does not directly work for arithmetic formulas. In order to construct a p-family in $\operatorname{VFC}\left(\leq_{c}\right) \backslash \operatorname{VFC}\left(\unlhd_{p}\right)$ we proceed as follows. Let $(f) \in \operatorname{VFC}\left(\leq_{c}\right)$, for example iterated $3 \times 3$ matrix multiplication. Define $(Q)$ via $Q_{n}:=\left(x+y f_{n}\right)^{2}$. We have $(Q) \notin \operatorname{VNPC}\left(\unlhd_{p}\right)$ in the same way as Lemma 7. Moreover, setting $x=0$ and $y=1$ we obtain $\beta_{1}:=f_{n}^{2}$. If instead we set $x=1$ and $y=1$, then we obtain $\beta_{2}:=f_{n}^{2}+2 f_{n}+1$. Hence $f_{n}=\frac{1}{2}\left(\beta_{2}-\beta_{1}-1\right)$. Thus $(f) \leq_{\mathrm{c}}(Q)$ and hence $(Q) \in \operatorname{VFC}\left(\leq_{c}\right)$. 


\section{The box topology is the correct topology}

In this section we prove that the closure of the classical classes VF, VBP, VP, and VNP in $R$ coincides with the classical definitions of $\overline{\mathrm{VF}}, \overline{\mathrm{VBP}}, \overline{\mathrm{VP}}$, and $\overline{\mathrm{VNP}}$ from the literature.

Given a polynomial $f$ with $v$ many variables and degree $d$. Let $V$ denote the finite dimensional vector space of polynomials of degree at most $d$ in $v$ many variables, endowed with the standard Euclidean metric $\rho$. Let $\operatorname{dc}(f)$ denote the determinantal complexity of $f$, i.e, the number of rows of the smallest square matrix $M$ with affine linear entries with $\operatorname{det}(M)=f$. Valiant proved that that $\operatorname{dc}(f)$ is always finite. Let $\underline{\operatorname{dc}}(f)$ denote the smallest $r$ such that there exists a sequence $\left(f_{m}\right)$ of polynomials with $\operatorname{dc}\left(f_{m}\right) \leq r$ such that $\lim _{m \rightarrow \infty} f_{m}=f$. The set of p-families $(f)$ where $\underline{\mathrm{dc}}((f))$ is polynomially bounded is called $\overline{\mathrm{VBP}}$ in the literature. We call it $\mathrm{VBP}^{\prime}$ here to make it possible to distinguish it from the closure of $\mathrm{VBP}$. We define $\mathrm{VF}^{\prime}, \mathrm{VP}^{\prime}$, and $\mathrm{VNP}^{\prime}$ analogously via their respective complete polynomials.

- Proposition 10. $\mathrm{VF}^{\prime}=\overline{\mathrm{VF}}$ and $\mathrm{VBP}^{\prime}=\overline{\mathrm{VBP}}$ and $\mathrm{VP}^{\prime}=\overline{\mathrm{VP}}$ and $\mathrm{VNP}^{\prime}=\overline{\mathrm{VNP}}$.

Proof. We only show the argument for VBP. The other cases are analogous.

Let $(f) \in \mathrm{VBP}^{\prime}$. This means that $\underline{\mathrm{dc}}((f))$ is polynomially bounded, say $\underline{\mathrm{dc}}((f))=p(n)$ for a polynomially bounded function $p$. Then for every $n$ and every $\varepsilon_{n} \in \mathbb{R}_{>0}$ there exists $g_{n, \varepsilon_{n}}$ with $\rho\left(f_{n}, g_{n, \varepsilon_{n}}\right)<\varepsilon_{n}$ and $\operatorname{dc}\left(g_{n, \varepsilon_{n}}\right) \leq p(n)$. Taking the downward directed set $D:=\left(\mathbb{R}_{>0}\right)^{\mathbb{N}}$ and setting $g_{n,(\varepsilon)}:=g_{n, \varepsilon_{n}}$, this defines a net $((g))$ in VBP. It remains to show that $((g))$ converges to $(f)$. Let $(\delta) \in D$. Then $\left(g_{(\delta)}\right) \in B_{(f),(\delta)}$ and for all $(\varepsilon) \preceq(\delta)$ we also have $\left(g_{(\varepsilon)}\right) \in B_{(f),(\delta)}$ by the definition of $g_{n,(\varepsilon)}$. Hence $((g))$ converges to $(f)$.

For the other direction we introduce a new concept. For every $(f) \in R$ we define a threshold distance sequence $\left(\delta^{\text {thresh }}\right) \in D$ by defining the $n$-th entry $\delta_{n}^{\text {thresh }}$ as follows. Let $v$ be the number of variables of $f_{n}$ and let $d$ be the degree of $f_{n}$. Let $V$ denote the finite dimensional vector space of polynomials of degree at most $d$ in $v$ many variables. Let $V_{r}:=$ $\{g \in V \mid \underline{\mathrm{dc}}(g) \leq r\}$. Each set $V_{r}$ is closed in $V$, in fact $V_{r}=\overline{\{g \in V \mid \operatorname{dc}(g) \leq r\}}$. We have $f_{n} \in V_{r}$ for all $r \geq \underline{\mathrm{dc}}(f)$, and $f_{n} \notin V_{r}$ otherwise. We define $\delta_{n}^{\text {thresh }}$ as the minimum distance between $f_{n}$ and $V_{r}$ for $r<\underline{\mathrm{dc}}(f)$, formally $\delta_{n}^{\text {thresh }}:=\min \left\{\rho\left(f_{n}, V_{r}\right) \mid r<\underline{\mathrm{dc}}(f)\right\}^{12}$. Here we use the usual definition of a distance of a point to a set: $\rho\left(f_{n}, V_{r}\right):=\inf \left\{\rho\left(f_{n}, g\right) \mid g \in V_{r}\right\}$. Note that $\delta_{n}^{\text {thresh }}>0$, because each $V_{r}$ is a closed set (a point has distance 0 to a closed set if and only if that set contains the point).

Let $(f) \in \overline{\mathrm{VBP}}$. Then there exists a downward directed set $(\mathcal{D}, \preceq)$ and a net $((g)): \mathcal{D} \rightarrow$ VBP that converges to $(f)$. Hence for every $(\delta) \in D$ there exists $\varepsilon \in \mathcal{D}$ such that for all $\varepsilon^{\prime} \preceq \varepsilon$ we have $\left(g_{\varepsilon^{\prime}}\right) \in B_{(f),(\delta)}$. In particular this holds for $\left(\delta^{\text {thresh }}\right)$, so $\left(g_{\varepsilon}\right) \in B_{(f),\left(\delta^{\text {thresh }}\right)}$ and $\operatorname{dc}\left(\left(g_{\varepsilon}\right)\right)$ is polynomially bounded. By the definition of $\delta^{\text {thresh }}$ it follows that for all $n$ we have $\underline{\mathrm{dc}}\left(f_{n}\right) \leq \mathrm{dc}\left(g_{n, \varepsilon}\right)$. Hence $\underline{\mathrm{dc}}((f))$ is polynomially bounded, i.e., $(f) \in \mathrm{VBP}^{\prime}$.

It is clear that this proof technique is not limited to VF, VBP, VP, and VNP, but it works for every algebraic complexity class that is defined by circuits of a specific kind growing at a specific rate.

\section{References}

1 Eric Allender and Fengming Wang. On the power of algebraic branching programs of width two. Comput. Complex., 25(1):217-253, March 2016.

\footnotetext{
${ }^{12}$ For determinantal complexity this equals $\rho\left(f_{n}, V_{\mathrm{dc}(f)-1}\right)$, but that is not important here.
} 
2 Michael Ben-Or and Richard Cleve. Computing algebraic formulas using a constant number of registers. SIAM Journal on Computing, 21(1):54-58, 1992.

3 D. Bini. Relations between exact and approximate bilinear algorithms. applications. CALCOLO, 17(1):87-97, Jan 1980.

4 Dario Bini, Grazia Lotti, and Francesco Romani. Approximate solutions for the bilinear form computational problem. SIAM Journal on Computing, 9(4):692-697, 1980.

5 Markus Bläser and Christian Ikenmeyer. Introduction to geometric complexity theory. http: //pcwww.liv.ac.uk/ iken/teaching_sb/summer17/introtogct/gct.pdf, 2018.

6 Markus Bläser, Christian Ikenmeyer, Meena Mahajan, Anurag Pandey, and Nitin Saurabh. Algebraic Branching Programs, Border Complexity, and Tangent Spaces. In Shubhangi Saraf, editor, 35th Computational Complexity Conference (CCC 2020), volume 169 of Leibniz International Proceedings in Informatics (LIPIcs), pages 21:1-21:24, Dagstuhl, Germany, 2020. Schloss Dagstuhl-Leibniz-Zentrum für Informatik.

7 Karl Bringmann, Christian Ikenmeyer, and Jeroen Zuiddam. On algebraic branching programs of small width. J. ACM, 65(5):32:1-32:29, 2018.

8 Peter Bürgisser. On the structure of Valiant's complexity classes. Discrete Mathematics and Theoretical Computer Science, 3(3):73-94, 1999.

9 Peter Bürgisser. The complexity of factors of multivariate polynomials. Found. Comput. Math., 4(4):369-396, 2004.

10 Peter Bürgisser, Joseph M Landsberg, Laurent Manivel, and Jerzy Weyman. An overview of mathematical issues arising in the geometric complexity theory approach to VP $\neq \mathrm{VNP}$. SIAM Journal on Computing, 40(4):1179-1209, 2011.

11 Don Coppersmith and Shmuel Winograd. On the asymptotic complexity of matrix multiplication. SIAM Journal on Computing, 11(3):472-492, 1982.

12 Don Coppersmith and Shmuel Winograd. Matrix multiplication via arithmetic progressions. Journal of Symbolic Computation, 9(3):251-280, 1990. Premliminary version in STOC 1987.

13 Pranjal Dutta, Nitin Saxena, and Amit Sinhababu. Discovering the roots: uniform closure results for algebraic classes under factoring. In Ilias Diakonikolas, David Kempe, and Monika Henzinger, editors, Proceedings of the 50th Annual ACM SIGACT Symposium on Theory of Computing, STOC 2018, Los Angeles, CA, USA, June 25-29, 2018, pages 1152-1165. ACM, 2018.

14 Michael A Forbes and Amir Shpilka. A PSPACE construction of a hitting set for the closure of small algebraic circuits. In Proceedings of the 50th Annual ACM SIGACT Symposium on Theory of Computing, pages 1180-1192, 2018.

15 Joshua A. Grochow, Ketan D. Mulmuley, and Youming Qiao. Boundaries of VP and VNP. In Ioannis Chatzigiannakis, Michael Mitzenmacher, Yuval Rabani, and Davide Sangiorgi, editors, $43 r d$ International Colloquium on Automata, Languages, and Programming (ICALP 2016), volume 55 of Leibniz International Proceedings in Informatics (LIPIcs), pages 34:1-34:14, Dagstuhl, Germany, 2016. Schloss Dagstuhl-Leibniz-Zentrum fuer Informatik.

16 Zeyu Guo, Nitin Saxena, and Amit Sinhababu. Algebraic dependencies and PSPACE algorithms in approximative complexity over any field. Theory of Computing, 15(1):1-30, 2019.

17 L.A. Hemaspaandra and Ogihara M. The Complexity Theory Companion. Springer, 2002.

18 John M. Hitchcock and A. Pavan. Comparing reductions to NP-complete sets. Information and Computation, 205(5):694 - 706, 2007.

19 Jesko Hüttenhain. Geometric complexity theory and orbit closures of homogeneous forms. 2017.

20 Christian Ikenmeyer and Stefan Mengel. On the relative power of reduction notions in arithmetic circuit complexity. Inf. Process. Lett., 130:7-10, 2018.

21 Christian Ikenmeyer and Abhiroop Sanyal. A note on VNP-completeness and border complexity (version 1). arXiv:2102.07173v1, 2021. 
22 Christian Ikenmeyer and Abhiroop Sanyal. A note on VNP-completeness and border complexity (version 2). arXiv:2102.07173v2, 2021.

23 Erich Kaltofen. Single-factor Hensel lifting and its application to the straight-line complexity of certain polynomials. In Alfred V. Aho, editor, Proceedings of the 19th Annual ACM Symposium on Theory of Computing, 1987, New York, New York, USA, pages 443-452. ACM, 1987.

24 John L Kelley. General topology. Courier Dover Publications, 2017.

25 Mrinal Kumar. On top fan-in vs formal degree for depth-3 arithmetic circuits. arXiv:1804.03303, 2018.

26 Joseph M Landsberg and Mateusz Michałek. A $2 \mathrm{n}^{2}-\log _{2} \mathrm{n}-1$ lower bound for the border rank of matrix multiplication. International Mathematics Research Notices, 2018(15):4722-4733, 03 2017.

27 Joseph M. Landsberg and Giorgio Ottaviani. New lower bounds for the border rank of matrix multiplication. Theory of Computing, 11(11):285-298, 2015.

28 Thomas Lehmkuhl and Thomas Lickteig. On the order of approximation in approximative triadic decompositions of tensors. Theoretical Computer Science, 66(1):1 - 14, 1989.

29 Meena Mahajan and Nitin Saurabh. Some complete and intermediate polynomials in algebraic complexity theory. Theory of Computing Systems, 62(3):622-652, 2018.

30 Dori Medini and Amir Shpilka. Hitting sets and reconstruction for dense orbits in $\mathrm{VP}_{e}$ and $\Sigma \Pi \Sigma$ circuits. In 36th Computational Complexity Conference (CCC 2021). Schloss Dagstuhl-Leibniz-Zentrum für Informatik, 2021.

31 K.D. Mulmuley and M. Sohoni. Geometric Complexity Theory I. An approach to the P vs. NP and related problems. SIAM J. Comput., 31(2):496-526, 2001.

32 Francesco Romani. Some properties of disjoint sums of tensors related to matrix multiplication. SIAM Journal on Computing, 11(2):263-267, 1982.

33 Ramprasad Saptharishi. A survey of lower bounds in arithmetic circuit complexity. Version 9.0.3, https://github.com/dasarpmar/lowerbounds-survey, 2021.

34 Arnold Schönhage. Partial and total matrix multiplication. SIAM Journal on Computing, 10(3):434-455, 1981.

35 Amit Kumar Sinhababu. Power series in complexity: Algebraic Dependence, Factor Conjecture and Hitting Set for Closure of VP. PhD thesis, PhD thesis, Indian Institute of Technology Kanpur, 2019.

36 Volker Strassen. Vermeidung von Divisionen. Journal für die reine und angewandte Mathematik, 264:184-202, 1973.

37 Volker Strassen. The asymptotic spectrum of tensors and the exponent of matrix multiplication. In 27th Annual Symposium on Foundations of Computer Science (sfcs 1986), pages 49-54. IEEE, 1986.

38 Seinosuke Toda. Classes of arithmetic circuits capturing the complexity of computing the determinant. IEICE Transactions on Information and Systems, 75(1):116-124, 1992.

39 Leslie G. Valiant. Completeness classes in algebra. In Conference Record of the Eleventh Annual ACM Symposium on Theory of Computing (Atlanta, Ga., 1979), pages 249-261. ACM, New York, 1979.

40 Leslie G. Valiant. Negation can be exponentially powerful. In Michael J. Fischer, Richard A. DeMillo, Nancy A. Lynch, Walter A. Burkhard, and Alfred V. Aho, editors, Proceedings of the $11 \mathrm{~h}$ Annual ACM Symposium on Theory of Computing, April 30 - May 2, 1979, Atlanta, Georgia, USA, pages 189-196. ACM, 1979. 